\title{
Clinical Prognostic Factors and Treatment Outcomes for the Survival of Patients with Cholangiocarcinoma in the Eastern Region of Thailand
}

\author{
Chanchai Charonpongsuntorn ${ }^{1}$, Piyanuch Piyasatit ${ }^{1}$, Dittapol Muntham², \\ Piyanant Chommaitree ${ }^{1}$, Panunat Muangnoi ${ }^{1}$
}

${ }^{1}$ Department of Internal Medicine, HRH Princess Maha Chakri Sirindhorn Medical Center, Faculty of Medicine, Srinakharinwirot University, Nakhon Nayok, Thailand. ${ }^{2}$ Department of Mathematics, Faculty of Science and Technology, Rajamangala University of Technology Suvarnabhumi, Thailand.

\begin{abstract}
Background: Cholangiocarcinoma (CCA) is an uncommon primary liver cancer worldwide but it commonly in Asia including Thailand. Although increasing incidence but the prognosis is poor and lethal cancer. Prognostic factors for CCA that affect survival outcomes are various due to a vary presentation and different diagnostic and therapeutic modalities. To date, satisfactory prognostic factors have not been understood. Objective: This study evaluated clinical prognostic factors and treatment that affect survival outcomes in patients with CCA. Methods: The CCA patients were identified from the database between January 2012 and December 2016. A retrospective review of 82 medical records was performed. Median follow time was more than 12 months. Survival outcome was estimated using Kaplan-Meier analysis. Demographic, clinical presentation, Laboratory, histopathological data and treatment modalities that affect survival were evaluated for prognostic factors relating to mortality by using univariate and multivariable statistical analysis. Result: 82 patients were identified. Median overall survival was 11.1 months. An unresectable or metastatic CCA patient was 74.4\% (61/82). Cox-regression univariate reports Higher Alkaline phosphatase level, Prolong Prothrombin time, Higher WBC count, Higher CA 19-9 level, Unresectable, Metastasis were identified as important clinical predictors of patient mortality. Treatment modalities factors that significantly affect survival outcome were Bile duct drainage, surgery, and palliative chemotherapy. A Poor prognostic outcome of survival depends on staging by resectability and metastasis $(\mathrm{P}<0.01)$. Conclusion: In this study identified that potential of surgery as the mainstay of treatment provided the best survival prognosis of patients with CCA. Treatment involving both bile duct drainage and chemotherapy also prolong CCA patient survival. Poor clinical prognostic factors which shorten survival are prolonging PT, higher WBC, ALP, and CA19-9.
\end{abstract}

Keywords: Cholangiocarcinoma- Prognostic factor- treatment outcomes- overall survival

Asian Pac J Cancer Care, 4 (4), 101-105

\section{Introduction}

Cholangiocarcinoma (CCA) is a malignancy arising from the epithelial cells of the bile duct in intrahepatic and extrahepatic [1]. It occurs with a rare disease in the United States and Europe region [2-4], but a greater prevalence and serious cancer in Thailand. Especially in Northeastern and North region, there is a high prevalence rate of biliary tract cancer (BTC) as 38.6 per 100,000 population, where over $80 \%$ of BTC was CCA [5]. The current systematic
Submission Date: 04/05/2019 Acceptance Date: 07/01/2019

review and meta-analysis indicated that the unique strong risk factors including Opisthorchis viverrini infection, eating raw cyprinoid fish, family history of cancer, liquor consumption, and taking praziquantel were increased risk of CCA in Thailand [6], but Hepatitis B, C infection, obesity and primary sclerosing cholangitis (PSC) are high-risk factors in the European population [7-8]. Moreover, the previous study found that the mortality rate was 16.9 per 100 person-months. The median survival time among CCA patients was 4.3 months. The

Department of Internal Medicine, HRH Princess Maha Chakri Sirindhorn Medical Center, Faculty of Medicine, Srinakharinwirot University,

Nakhon Nayok, Thailand.

Email: Chanchaic@g.swu.ac.th 
independent factors such as the stage of the disease, presentation with jaundice or ascites, positive serum carcinoembryonic antigen were related with poor survival time, however alternative medicine was increased with survival time as $50 \%$ of CCA patients [9]. Therefore, Survival time of CCA patients can be improved by early detection followed by the best method curative resection, but only $25 \%$ of patients are eligible for curative surgery because they usually present at metastases or late stage of the disease [10-11]. However, the previous studies indicated that the patients in the chemotherapy had an improved or prolonged high quality of life more than the best supportive care group [12]. At the current study, there are 2 the best chemotherapy (5-FU Based regimen and Gemcitabine Based regimen) for CCA remains to be used in Thailand. Data from several studies suggest that 5-FU Based regimen was overall response rate 10-40\% and overall median survival about 2-12 months [13-14]. Meanwhile, the gemcitabine-based regimen was overall response rate $8-50 \%$ and overall median survival about 5-15.40 months [13-16]. According to the randomized controlled trial phase, III (ABC-02) suggests that the median overall survival was higher in among the patients in the gemcitabine plus cisplatin group than the patients in the gemcitabine alone (11.7 months vs. 8.1 months, respectively) [16]. However, some previous study found that there was no difference between overall survival with the use of gemcitabine plus cisplatin (15.2 months) or alternative regimens (13.9 months) [17], in similarly previous results found that the survival of patient with advanced CCA treated with gemcitabine-based and 5FU-based chemotherapy also do not markedly differ [18]. Therefore, the role of 5-FU and gemcitabine-based regimen also needs further study. Further investigation of new chemotherapeutic agents and novel targeted therapy is warranted.

To our knowledge, there is no evidence demonstrated that the efficacy of chemotherapy, best supportive care and curative surgery on survival among CCA patients in Eastern region of Thailand. Thus, the present study aimed to investigate the survival rates and factors affecting survival in patients with CCA patients at HRH Princess Maha Chakri Sirindhorn Medical Center (MSMC) during 2012-2017. The outcomes are expected to be useful for improving the treatment modality and the quality of life of patients.

\section{Materials and Methods}

\section{Study design and study population}

A retrospective cohort study was collected with 82 CCA patients, who were diagnosed by a physician as CCA at least one of the following diagnosed criteria: symptomatic, imaging or pathological findings, age over 18 years and treated by chemotherapy, best supportive care and curative surgery during the period 1 January 2012 to 31 December 2012 at HRH Princess Maha Chakri Sirindhorn Medical Center (MSMC), Thailand. we excluded CCA patients with others cancer, metastatic $\mathrm{CCA}$, and missing clinical variables such as liver function test (LFT), hepatitis B virus surface antigen (HBsAg), carcinoembryonic antigen (CEA), serum carbohydrate antigen 19-9 (CA19-9), and antibodies against hepatitis C virus (anti-HCV). The CCA patients were follow-up until death or the end of study (31 December 2017).

\section{Data collection}

Patient baseline demographics such as gender, age at recruitment, residence, occupation, health insurance, underlying disease, lifetime alcohol history, lifetime smoking history, and cancer family history were recorded through review of medical records. Past clinical history data including any clinical symptom, body mass index, Eastern cooperative oncology group (ECOG) score, duration of preclinical, type of CCA, stage of disease and metastasis was determined by review of clinical charts. Laboratory including CBC, LFT, CEA, CA19-9, $\mathrm{HBsAg}$, Anti-HCV, and imaging results at the time of initial diagnosis was included. Moreover, Information on the type of treatment (chemotherapy, best supportive care, and surgery) and response evaluation criteria in solid tumors (RECIST) were also included in our study analysis.

\section{Outcome measurement}

The dependent variable was the survival time of patients with CCA. The authors calculate the date of CCA diagnosis and the follow-up until death or the end of the study on 31 December 2017. The death status of each CCA patients was checked from medical records and confirmed by linkage with the death certificates from the national statistics database as well as by a telephone call to the patients or public health officers in the health-promoting hospital (HPH). Moreover, the censored data were defined as alive at the end of the study, or death unrelated to CCA during the study period or lost to follow-up.

\section{Statistical analyses}

The baseline characteristics, laboratory, and clinical subject data were presented as descriptive statistics. Frequency and percentages were used to describe categorical data and means with standard deviations or medians with ranges were used to describe continuous data. The observed survival rate was calculated by the Kaplan-Meier method. Median survival times with 95\% confidence intervals (CIs) and the log-rank test were used for comparisons between groups. The univariate and multivariate Cox proportional hazard regression model was used to assess associations between the various covariates and survival. The results are presented as hazard ratios (HR) and 95\% confidence intervals $(95 \% \mathrm{CI})$. The level of statistical significance was set as a p-value less than 0.05 .

\section{Results}

Figure 1 showed the type of treatments given to patients. Approximately 41 of all patients received with best supportive care (BSC). In total, 22 patients were treated with surgical resection. Finally, there were 19 patients who were treated with Chemotherapy. The median 
Table 1. Univariate and Multivariate Analysis of Predictors of Survival in Patients with CCA

\begin{tabular}{|c|c|c|c|c|c|c|}
\hline \multirow[t]{2}{*}{ Factors } & \multicolumn{3}{|c|}{ Univariate } & \multicolumn{3}{|c|}{ Multivariate } \\
\hline & HR & $95 \% \mathrm{CI}$ & P-value & $\mathrm{HR}$ & $95 \% \mathrm{CI}$ & P-value \\
\hline Alkaline phosphatase (>126 IU/L) & 2.23 & $1.09-4.54$ & 0.01 & & & \\
\hline Prothrombin time $(>13)$ & 1.72 & $1.00-3.05$ & 0.05 & & & \\
\hline WBC (>10,000/dL) & 1.72 & $1.04-2.84$ & 0.03 & & & \\
\hline CA $19-9$ (>300 ng/mL) & 3.03 & $1.66-5.53$ & $<0.01$ & & & \\
\hline Unresectability & 2.49 & $1.23-5.05$ & $<0.01$ & 3.70 & $1.54-8.89$ & $<0.01$ \\
\hline Metastasis & 2.95 & $1.44-6.02$ & $<0.01$ & 7.47 & $2.88-19.47$ & $<0.01$ \\
\hline Bile duct drainage (PTBD/Stent) & 2.11 & $1.27-3.50$ & $<0.01$ & & & \\
\hline Surgery & 0.24 & $0.12-0.50$ & $<0.01$ & & & \\
\hline Palliative chemotherapy & 0.55 & $0.31-0.99$ & 0.05 & & & \\
\hline
\end{tabular}

HR, Hazard ratio; CI, Confidence interval; CEA, carcinoembryonic antigen; CA 19-9, serum carbohydrate antigen 19-9; WBC, White blood cell count; CCA, Cholangiocarcinoma.

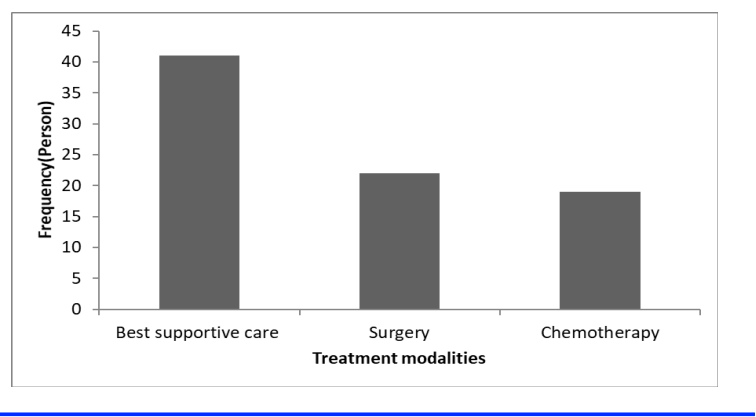

Figure 1. Type of Treatment

survival of the 82 patients with CCA after diagnosis was 11.1 months (Figure 2). Moreover, the median overall survival time was 5.12 months for BSC patients, 13.38 months for chemotherapy patients, especially, the median overall survival time was high (29.38 months) for surgical resection patients. The difference in survival times was statistically significant (log-rank test P-value $<0.01$ ) as shown in Figure 3.

From the analyses to investigate the factors associated with the survival time of CCA patients. The univariate analysis found that bile duct drainage, surgery, palliative chemotherapy, and several clinical factors including higher alkaline phosphatase level, prolong prothrombin time, higher WBC count, positive CA 19-9 level, unresectable, metastasis was statistically significant $(p$-value $<0.05)$ association with survival time. In the results of the multivariable analysis, using Cox's proportional hazard

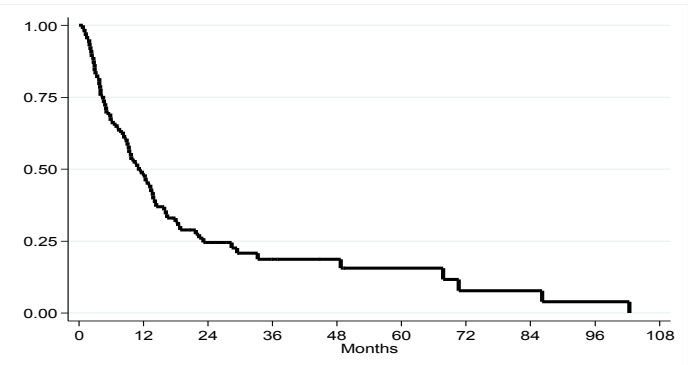

Figure 2. Kaplan-Meier Curve of Overall Median Survival Time in CCA Patients $(\mathrm{N}=82)$ model as shown in Table 1 . The results showed that only unresectable (HR: 3.70, 95\%CI: 1.54-8.89) and metastasis (HR: 7.47, 95\%CI: 2.88-19.47) was statistically significant (p-value $<0.05)$.

\section{Discussion}

In the present study, we reported the impact of three types of treatment modalities. Surgery was the most effective treatment, with an overall survival time of 29.38 months. The overall survival times are consistent with the findings of previous studies [19]. Differences from the findings of previous studies might due to patient characteristics and length of follow-up. However, the previous studies indicated that the best method curative resection can be increased the overall survival times in CCA patients, only $25 \%$ of CCA patients are eligible for curative surgery because they usually present at metastases or late stage of the disease [10-11]. Meanwhile, the median overall survival time was 13.38 and 5.12 months for chemotherapy and BSC patients, respectively. The several previous studies supported that chemotherapy treatment had an improved or prolonged high quality of life more than the BSC [12]. At the previous studies has already been shown to be effective therapy such as 5-FU based regimen and gemcitabine-based regimen for CCA patients. 5-FU Based regimen was overall response rate $10-40 \%$ and overall median survival about 2-12 months [13-14]. Meanwhile, the gemcitabine-based regimen

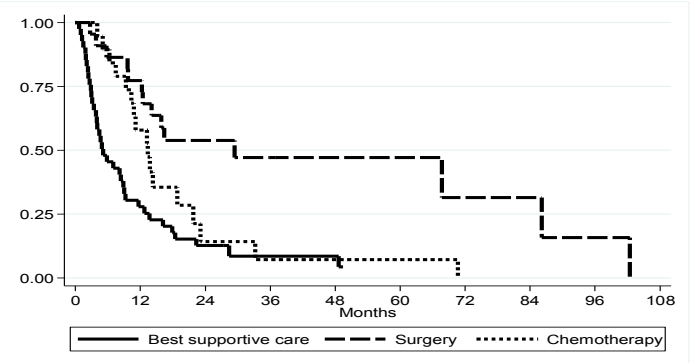

Figure 3. Kaplan-Meier Curve of Overall Median Survival Time in CCA Patients According to Treatment Modalities 
was overall response rate $8-50 \%$ and overall median survival about 5-15.40 months [13-16]. According to the randomized controlled trial phase, III (ABC-02) showed that gemcitabine plus cisplatin regimen is an appropriate option for the treatment of advanced CCA patients [16]. However, the survival of a patient with advanced CCA treated with gemcitabine-based and 5FU-based chemotherapy also do not markedly differ [18].

In the univariate analysis, The bile duct drainage, surgery, palliative chemotherapy unresectable, metastasis, and several clinical factors including higher alkaline phosphatase level, prolonged prothrombin time, higher WBC count, positive CA 19-9 level were associated with survival rate. These findings are similar to those reported in previous studies [9-20]. However, in our multivariate analysis, metastasis had 7.47 times $(95 \% \mathrm{CI}$ : 2.88-19.47) increased the risk of poor survival and those with unresectable had 3.70 times (95\%CI: $1.54-8.89)$ also increased the risk of poor survival rates of CCA patients. These findings are almost different as those in the findings of previous studies [9-19-20]; the patients with CA 19-9 levels, bilirubin level, stage of disease presentation with jaundice, and ascites had lower survival rates. Differences from the findings of previous studies might due to patient characteristics, variables affecting treatment and survival outcomes, such as the numbers of samples, the duration of the study, and physician and patient post diagnosis status. Whereas, our results found that surgery and palliative chemotherapy has no statistically significant prognostic factors affecting survival. These findings are almost the same as the study reported by [21]. However, some reported a rather different finding; alternative medicine was increased with survival time as $50 \%$ of CCA patients [9].

This study is the first study in the Eastern region of Thailand, but there was some limitation. Firstly, the limitations of this study are its single, tertiary-care institution retrospective study and its nonrandomized design, which can introduce selection bias and make the results less generalizable. Secondary, the sample size was not large, making sub-group analysis difficult, and there were some unbalances of baseline information in patient characteristics between the treatment groups. In addition, most lost cases were patients who have missing values such as laboratory results and staging. Finally, our present study not collected some covariate factors such as quantification of alcohol and tobacco use, and co-morbidities.

In summary, the median overall survival of the CCA patients after diagnosis was 11.1 months in the Eastern region of Thailand. Surgical resection is the most effective treatment, with higher overall survival than chemotherapy and BSC. The prognostic factors effect of survival in our cohort included metastasis and unresectable. There were no significant differences between treatment options and overall survival. Further randomized trials studies with relatively large samples are required with an emphasis on strong prognostic factors such as a type of cancer, performance status, and presence of metastases, which may help in CCA prognosis in Thais population.

\section{Acknowledgments}

The authors would like to thank all colleagues in the Department of Internal Medicine, Faculty of Medicine, Srinakharinwirot University especially the Hepatocellular excellent center team for their invaluable help and encouragement throughout the course of this research. Finally, the authors were most appreciated for the participation of all patients in this research.

\section{Funding Statement}

The authors received funding for this work from the faculty of medicine, Srinakharinwirot University.

\section{References}

1. Nakeeb A, Pitt HA, Sohn TA, Coleman J, Abrams RA, Piantadosi S, et al. Cholangiocarcinoma. A spectrum of intrahepatic, perihilar, and distal tumors. Ann Surg. 1996;224(4):463-73; discussion 73-5.

2. de Groen PC, Gores GJ, LaRusso NF, Gunderson LL, Nagorney DM. Biliary tract cancers. The New England journal of medicine. 1999;341(18):1368-78.

3. Jemal A, Bray F, Center MM, Ferlay J, Ward E, Forman D. Global cancer statistics. CA: a cancer journal for clinicians. 2011;61(2):69-90.

4. Bray F, Ferlay J, Soerjomataram I, Siegel RL, Torre LA, Jemal A. Global cancer statistics 2018: GLOBOCAN estimates of incidence and mortality worldwide for 36 cancers in 185 countries. CA: a cancer journal for clinicians. 2018;68(6):394-424.

5. Banales JM, Cardinale V, Carpino G, Marzioni M, Andersen JB, Invernizzi P, et al. Expert consensus document: Cholangiocarcinoma: current knowledge and future perspectives consensus statement from the European Network for the Study of Cholangiocarcinoma (ENSCCA). Nature reviews Gastroenterology \& hepatology. 2016;13(5):261-80

6. Kamsa-ard S K-aS, Luvira V, et al. Risk Factors for Cholangiocarcinoma in Thailand: A Systematic Review and Meta-Analysis. Asian Pacific journal of cancer prevention : APJCP. 2018;19:605.

7. Chapman RW. Risk factors for biliary tract carcinogenesis. Annals of oncology : official journal of the European Society for Medical Oncology. 1999;10 Suppl 4:308-11.

8. Watanapa P, Watanapa WB. Liver fluke-associated cholangiocarcinoma. The British journal of surgery. 2002;89(8):962-70.

9. Woradet S, Promthet S, Songserm N, Parkin DM. Factors affecting survival time of cholangiocarcinoma patients: a prospective study in Northeast Thailand. Asian Pacific journal of cancer prevention : APJCP. 2013;14(3):1623-7.

10. Anderson CD, Pinson CW, Berlin J, Chari RS. Diagnosis and treatment of cholangiocarcinoma. The oncologist. 2004;9(1):43-57.

11. Vogel A, Wege H, Caca K, Nashan B, Neumann U. The diagnosis and treatment of cholangiocarcinoma. Deutsches Arzteblatt international. 2014;111(44):748-54.

12. Glimelius B, Hoffman K, Sjoden PO, Jacobsson G, Sellstrom $\mathrm{H}$, Enander LK, et al. Chemotherapy improves survival and quality of life in advanced pancreatic and biliary cancer. Annals of oncology : official journal of the European Society for Medical Oncology. 1996;7(6):593-600.

13. Thongprasert S. The role of chemotherapy in cholangiocarcinoma. Annals of oncology : official journal of the European Society for Medical Oncology. 2005;16 
Suppl 2:ii93-6.

14. Hezel AF, Zhu AX. Systemic therapy for biliary tract cancers. The oncologist. 2008;13(4):415-23.

15. Charoentum C, Thongprasert S, Chewaskulyong B, Munprakan S. Experience with gemcitabine and cisplatin in the therapy of inoperable and metastatic cholangiocarcinoma. World journal of gastroenterology. 2007;13(20):2852-4.

16. Valle J, Wasan H, Palmer DH, Cunningham D, Anthoney A, Maraveyas A, et al. Cisplatin plus gemcitabine versus gemcitabine for biliary tract cancer. The New England journal of medicine. 2010;362(14):1273-81.

17. Eckmann KR, Patel DK, Landgraf A, Slade JH, Lin E, Kaur H, et al. Chemotherapy outcomes for the treatment of unresectable intrahepatic and hilar cholangiocarcinoma: a retrospective analysis. Gastrointestinal cancer research : GCR. 2011;4(5-6):155-60.

18. Butthongkomvong K, Sirachainan E, Jhankumpha S, Kumdang S, Sukhontharot OU. Treatment outcome of palliative chemotherapy in inoperable cholangiocarcinoma in Thailand. Asian Pacific journal of cancer prevention : APJCP. 2013;14(6):3565-8.

19. Pattanathien P, Khuntikeo N, Promthet S, Kamsa-Ard S. Survival rate of extrahepatic cholangiocarcinoma patients after surgical treatment in Thailand. Asian Pacific journal of cancer prevention : APJCP. 2013;14(1):321-4.

20. Singal AG, Rakoski MO, Salgia R, Pelletier S, Welling TH, Fontana RJ, et al. The clinical presentation and prognostic factors for intrahepatic and extrahepatic cholangiocarcinoma in a tertiary care centre. Alimentary pharmacology \& therapeutics. 2010;31(6):625-33.

21. Thunyaharn N, Promthet S, Wiangnon S, Suwanrungruang K, Kamsa-ard S. Survival of cholangiocarcinoma patients in northeastern Thailand after supportive treatment. Asian Pacific journal of cancer prevention : APJCP. 2013;14(11):7029-32.

c) () (8)

This work is licensed under a Creative Commons AttributionNon Commercial 4.0 International License. 